\title{
Sleep quality, neurocognitive performance, and memory self-appraisal in middle-aged and older adults with memory complaints
}

\author{
Prabha Siddarth, ${ }^{1,2,3}$ Kitikan Thana-udom, ${ }^{1,4}$ Rashi Ojha, 1,2,3 David Merrill,, \\ Joseph M. Dzierzewski, ${ }^{6}$ Karen Miller, ${ }^{1,2,3}$ Gary W. Small, ${ }^{1,2,3}$ and Linda Ercoli,2,3 \\ ${ }^{1}$ Semel Institute for Neuroscience and Human Behavior, UCLA, Los Angeles, CA, USA \\ ${ }^{2}$ Longevity Center, David Geffen School of Medicine, UCLA, Los Angeles, CA, USA \\ ${ }^{3}$ Division of Geriatric Psychiatry, Department of Psychiatry and Biobehavioral Sciences, UCLA, Los Angeles, CA, USA \\ ${ }^{4}$ Department of Psychiatry, Faculty of Medicine Siriraj Hospital, Mahidol University, Bangkok, Thailand \\ ${ }^{5}$ Pacific Brain Health Center at Pacific Neuroscience Institute, Santa Monica, CA, USA \\ ${ }^{6}$ Department of Psychology, Virginia Commonwealth University, Richmond, VA, USA
}

ABSTRACT

Objective: Because of inconsistent findings regarding the relationship between sleep quality and cognitive function in people with age-related memory complaints, we examined how self-reports of sleep quality were related to multiple domains of both objective and subjective cognitive function in middle-aged and older adults.

Design: A cross-sectional study involving analysis of baseline data, collected as part of a clinical trial.

Measurements: Two hundred and three participants (mean age $=60.4$ [6.5] years, 69.0\% female) with mild memory complaints were asked to rate their sleep quality using the Pittsburgh Sleep Quality Index (PSQI) and their memory performance using the Memory Functioning Questionnaire (MFQ), which measures selfawareness of memory ability. Neurocognitive performance was evaluated using the Continuous Performance Test (CPT), Trail Making Test, Buschke Selective Reminding Test, and the Brief Visuospatial Test - Revised (BVMT-R).

Results: Total PSQI scores were significantly associated with objective measures of sustained attention (CPT hit reaction time by block and standard error by block) and subjective memory loss (MFQ frequency and seriousness of forgetting). The PSQI components of (poorer) sleep quality and (greater) sleep disturbance were related to (worse) sustained attention scores while increased sleep latency and daytime sleepiness were associated with greater frequency and seriousness of forgetting.

Conclusions: Sleep quality is related to both objective measures of sustained attention and self-awareness of memory decline. These findings suggest that interventions for improving sleep quality may contribute not only to improving the ability to focus on a particular task but also in reducing memory complaints in middle-aged and older adults.

Key words: sleep quality, age-related cognitive decline, memory complaints

\section{Introduction}

Considerable evidence indicates the benefits of sufficient and high-quality sleep for physical and mental health, including cognition. Although the nuances of sleep patterns may change during a person's

Correspondence should be addressed to: Prabha Siddarth, Semel Institute for Neuroscience and Human Behavior, David Geffen School of Medicine at the University of California, Los Angeles, 760 Westwood Plaza, Los Angeles, CA 90095, USA. Phone: (310) 825-4295; Fax: (310) 825-3910. Email: PSiddarth@mednet.ucla.edu. Received 02 Jun 2020; revision requested 09 Jul 2020; revised version received 27 Jul 2020; accepted 15 Aug 2020. First published online 28 Sep 2020 lifetime (Ohayon et al., 2004), sleep remains critical for well-being at all stages of life. Some of the changes in sleep patterns that can occur in older adults include a reduction in total sleep time, decreased sleep efficiency, decreased quantity of slow wave and REM sleep, increased sleep fragmentation, and increased awakenings after sleep onset (Campbell and Murphy, 2007; Dijk et al., 2001; Dorffner et al., 2015; Ohayon et al., 2004; Scullin and Bliwise, 2015; Wolkove et al., 2007). Further, older adults report worse sleep quality compared to younger people (Crowley, 2011). 
In addition to changes in sleep patterns, aging is also associated with changes in cognition, which can include a decline in memory, information processing speed, attention and executive function (Davis et al., 2013; Salthouse, 2010), and both the quantity and quality of sleep may be a moderator for cognitive aging (Loerbroks et al., 2010). Longitudinal data show that adequate and high-quality sleep promotes cognitive health (Dzierzewski et al., 2018; Vance 2012), while increased sleep fragmentation and sleep disruption in older adults is associated with a higher rate of cognitive decline over a 3- to 5-year follow-up (Blackwell et al., 2014; Lim et al., 2013; McSorley et al., 2019).

Other studies that have examined the relationship between sleep problems and cognitive function in older adults have yielded inconsistent results. Some cross-sectional studies have not shown associations between subjective sleep quality and global cognition (Saint Martin et al., 2012) or any domains of cognition (Blackwell et al., 2011; Miyake et al., 2000), whereas other cross-sectional investigations have indicated an association between low-quality sleep and worsening of at least one component of cognitive performance, such as decline in processing speed (Bastien et al., 2003), decreased executive function (Nebes et al., 2009; Waters and Bucks, 2011), and impaired episodic memory (Tsapanou et al., 2017). Such inconsistencies may be attributed to differences in controlled variables, such as depressive symptoms, anxiety, or sleep medication use, or the inclusion of heterogeneous populations across studies, such as the inclusion of neurological and psychiatric comorbidities in participants (Bastien et al., 2003; Foley et al., 2003; Moore et al., 2001). Sample heterogeneity is particularly relevant because each domain of cognitive function has an intricate set of multiple sub-processes (Glisky, 2007) that may be uniquely linked to sleep quality in different populations. The lack of a uniform set of cognitive measures across studies makes it challenging to compare findings. In addition to the objective measures of cognition, subjective memory complaints, which can occur in the absence of objective cognitive decline, may predict future cognitive impairment and dementia (Choe et al., 2018; Jessen, 2010). Only a few studies, however, have examined the link between sleep quality and subjective memory complaints (Gamaldo et al., 2019; Kang et al., 2017), and these have indicated a possible connection between poor sleep and memory dysfunction.

A few imaging studies have investigated the effect of sleep quality on alterations in brain structure in older adults. In adults 55 years and older, selfreported short sleep duration was associated with greater age-related brain atrophy and cognitive decline 2 years later (Lo et al., 2014). Worse selfreported sleep was also found to be associated with greater hippocampal volume reduction across the adult lifespan (Fjell et al., 2020); however, the effect sizes (ES) were small. Thus, an understanding of how sleep quality may affect brain structure and cognition remains limited.

Given the lack of consistent findings in the literature with respect to sleep quality and neurocognitive performance and the paucity of research with respect to sleep quality and subjective memory complaints, we examined the association between subjective sleep measures and specific aspects of neurocognitive performance and self-reported memory in non-demented middle-aged and older adults with subjective memory complaints. We investigated four domains of objective cognition simple attention, sustained attention, memory recall, and executive function, and two aspects of subjective memory performance - frequency of forgetting and seriousness of forgetting. We anticipated that sustained attention, memory recall, executive function, and the two self-report memory measures would show significant impairment with poorer sleep quality, while simple attention was less likely to be affected.

\section{Methods}

\section{Participants}

The present study is a secondary analysis of baseline data collected on 221 middle-aged and older adults, who participated in a randomized, placebocontrolled trial exploring the cognitive effects of daily consumption of pomegranate juice (Identifier: NCT02093130; Siddarth et al., 2020) approved by the University of California, Los Angeles, Institutional Review Board. Participants were recruited through advertisements, media, and referrals from physicians and families. Our study protocol detailed the methods and procedures, as well as pre-specified inclusion and exclusion criteria. Exclusion criteria included participants with a diagnosis of Alzheimer's disease or other dementia, a major psychiatric or neurological disorder (e.g. major depressive disorder, schizophrenia, Parkinson's disease, Huntington's disease), alcohol or substance abuse, or a history of head trauma or systemic disease affecting brain function (e.g. systemic lupus erythematosus, congestive heart failure). Inclusion criteria were: (1) subjective memory complaints (initially assessed during a phone screen by an affirmative response to the question, "Are you now experiencing or have you experienced memory problems over the past 6 months?" and confirmed by responses to the 
Table 1. Baseline demographic and clinicalcharacteristics

\begin{tabular}{|c|c|c|}
\hline Characteristics & & $\operatorname{MeAN}(\mathrm{SD}) / \mathrm{n}(\%)$ \\
\hline \multicolumn{3}{|l|}{ Demographics } \\
\hline Age (years) & & $60.4(6.5)$ \\
\hline Gender ${ }^{\mathrm{a}}$ & Female & $140(69.0)$ \\
\hline \multirow[t]{4}{*}{ Ethnicity $^{\mathrm{a}}$} & Caucasian & $141(69.5)$ \\
\hline & Asian & $22(10.8)$ \\
\hline & Hispanic & $20(9.9)$ \\
\hline & Black & $15(7.4)$ \\
\hline Marital status ${ }^{\mathrm{a}}$ & Married & $119(58.6)$ \\
\hline Education (years) & & $16.4(2.1)$ \\
\hline ApoE4 status $(\mathrm{n}=189)$ & E4 allele carrier & $46(24.3)$ \\
\hline \multicolumn{3}{|l|}{ Clinical measures } \\
\hline Montreal Cognitive Assessment & & $27.7(1.5)$ \\
\hline Beck Depression Inventory ${ }^{\mathrm{b}}$ & & $4(0-18)$ \\
\hline STOP-BANG questionnaire (sleep apnea risk) & High risk of sleep apnea & $52(25.6)$ \\
\hline Total Pittsburgh Sleep Quality Index (PSQI) & & $4.9(2.9)$ \\
\hline PSQI component 1: Subjective sleep quality ${ }^{\mathrm{a}}$ & Bad quality & $29(14.3)$ \\
\hline PSQI component 2: Sleep latency ${ }^{\mathrm{a}}$ & $>15$ minutes & $116(57.1)$ \\
\hline PSQI component 3: Sleep duration ${ }^{a}$ & $\leq 7$ hours & $94(46.3)$ \\
\hline PSQI component 4: Habitual sleep efficiency & $\leq 85 \%$ & $53(26.1)$ \\
\hline PSQI component 5: Sleep disturbances ${ }^{\mathrm{a}}$ & More disturbances & $54(26.6)$ \\
\hline PSQI component 6: Use of sleep medication ${ }^{\mathrm{a}}$ & Used this week & $45(22.2)$ \\
\hline PSQI component 7: Daytime dysfunction ${ }^{\mathrm{a}}$ & Problem with sleepiness & $106(52.2)$ \\
\hline
\end{tabular}

${ }^{a}$ reported as $\mathrm{n}(\%)$.

${ }^{\mathrm{b}}$ reported as median score (range).

Memory Functioning Questionnaire [MFQ]); (2) aged 50 or greater; (3) no significant cerebrovascular disease defined as Stroke Risk Factor Prediction $<4$ (Wolf et al., 1991); (4) normal cognition, defined as Montreal Cognitive Assessment (MOCA) $\geq 25$ (Nasreddine et al., 2005); and (5) absence of significant depressive symptomology, defined as Beck Depression Inventory-II (BDI-II) $<18$ (Beck et al., 1996). Participants underwent neuropsychological and laboratory testing, including APOE genotyping. Written informed consent was obtained from all subjects in accordance with the University of California, Los Angeles Human Subjects Protection Committee procedures. The trial began in January 2014 and was completed in May 2018, and all data were collected at the Semel Institute for Neuroscience and Human Behavior and Center for Human Nutrition, David Geffen School of Medicine at the University of California, Los Angeles, CA. All data used in the present study were collected at baseline before the intervention was begun.

\section{Measures}

SleEP QUALITY

Sleep data were obtained using a self-report questionnaire, the Pittsburgh Sleep Quality Index
(PSQI). The PSQI assesses sleep quality exploring seven components of sleep. Each item has a range of 0 to -3 , and the seven items are summed for a Total PSQI score (lower scores indicating better sleep quality). With a cut-off score of 5, a PSQI total has a sensitivity of $89.6 \%$ and specificity of $86.5 \%$ for identifying disordered sleep (Buysse et al., 1989). The Total PSQI score was used as a continuous variable in analyses. In addition, we also examined the seven component scores. The distribution of these item scores did not allow their use as continuous variables in analyses, and hence they were dichotomized as below, depending on their distribution, such that there were sufficient participants in each group (see Table 1):

1. Subjective sleep quality: Good (score $0-1$ ) versus bad (score 2-3);

2. Sleep latency: Less than or equal to 15 minutes (score 0 ) versus more than 15 minutes (score 1-3);

3. Sleep duration: More than 7 hours (score 0 ) versus 7 hours or less (score 1-3);

4. Habitual sleep efficiency: more than $85 \%$ (score 0 ) versus $85 \%$ or less (score $1-3$ );

5. Sleep disturbance (i.e. sleep fragmentation or sleep disruption): Less disturbed (score $0-1$ ) versus more disturbed (score 2-3); 
6. Use of sleeping medication: No use (score 0) versuss use (score 1-3);

7. Daytime dysfunction: No problem (score 0) versus problem with daytime dysfunction (score 1-3).

Obstructive SLeEP apNea (OSA)

In addition to the PSQI, the STOP-BANG questionnaire (Chung et al., 2016) was used to screen for OSA. This instrument assesses four subjective items and four clinical items: S - "Do you snore loudly?"; $\mathrm{T}$ - "Do you often feel tired, fatigued, or sleepy during daytime?"; $\mathrm{O}$ - "Has anyone observed you stop breathing during your sleep?"; and P - "Do you have or are you being treated for high blood pressure?" The BANG part of the questionnaire consists of four demographic questions associated with risk for OSA: B - increased body mass index (over $35 \mathrm{~kg} / \mathrm{m} 2$ ); $\mathrm{A}$ - aged over 50 years; $\mathrm{N}$ - neck circumference over $40 \mathrm{~cm}$; and $\mathrm{G}$ - male gender. For each question, answering "yes" scores 1 , while a "no" response scores 0 , resulting in a range of $0-8$. A standard cut-off score of 3 is usually taken to indicate high risk for OSA.

\section{OBJECTIVE MEASURES OF COGNITIVE FUNCTIONING}

Within 1 month of the PSQI assessment, participants underwent a full neuropsychological assessment that focused on four domains: (1) Simple attention: measured using the five $\mathrm{T}$-scores of the Continuous Performance Test (CPT) (Conners, 2000): detectability, hit reaction time, standard error, reaction time by interstimulus interval, and standard error by interstimulus interval; (2)

Sustained attention (ability to maintain focus on a specific task for an extended period of time) (Fortenbaugh et al., 2017) measured by hit reaction time by block and standard error by block (Conners, 2000); (3) Executive function: this domain included two major components of executive function, namely cognitive set-shifting and the ability to inhibit responses. Set-shifting ability (adjusted for processing speed) was measured by subtracting the Trail Making Test A time score from Trail Making Test B time score (Arbuthnott and Frank, 2000). Inhibition was measured using $\mathrm{T}$-scores of commission errors, and hit reaction time of CPT; high $\mathrm{T}$-scores for both commission errors and hit reaction times are indicators of impulsivity (Conners, 2000). and (4) Recall memory: measured using the delayed recall score of Buschke-Fuld Selective Reminding Test (SRT) (Buschke, 1973) and the delayed recall score of the Brief Visual Memory Test - Revised (BVMT-R) (Benedict et al., 1996). $\mathrm{T}$-scores and raw scores were converted to $\mathrm{z}$-scores using the overall sample mean and standard deviation. For variables in which better performance was represented by lower values (e.g. T-scores of CPT, timed scores of the Trail Making Test), z-scores were reversed so that a higher score indicated better performance. A domain $\mathrm{z}$-score was obtained by averaging $\mathrm{z}$-scores.

SubJective ASSESSMENT OF MEMORY FUNCTIONING

Participants completed MFQ (Gilewski et al., 1990); two factor scores were used in the analysis from this 64-item questionnaire: frequency of forgetting (MFQ1) and seriousness of forgetting (MFQ2). Higher scores reflect higher levels of self-perceived memory functioning. Factor structure is stable across age groups and internal consistency is high, with Cronbach's alpha values for its factor scores ranging from 0.83 to 0.94 (Zelinski et al., 1990). The MFQ shows moderate concurrent validity with objective memory performance (Zelinski et al., 1990).

\section{Data analysis}

Prior to analyses, all data were screened for outliers and normality assumptions. Baseline clinical characteristics were described using total number (percent) for categorical data and mean (standard deviation) for continuous data. A separate general linear model was estimated to examine if sleep quality measured by Total PSQI was significantly associated with objective cognitive performance in each of the four domains, controlling for age, gender, education level, and baseline MOCA score. We also examined whether age, gender, OSA risk, and APOE-4 status moderated the relationship between cognitive functioning and sleep, by including the interactions in turn (age $\times$ PSQI; gender $\times$ PSQI; OSA risk $\times$ PSQI, ApoE $4 \times$ PSQI) in the models. The moderating effects of age, gender, OSA risk, and ApoE4 were tested since all these variables are known to affect sleep and/or cognitive performance (Gosselin et al., 2019; Krishnan and Collop, 2006; Lim et al., 2013; Scullin and Bliwise, 2015). Similar general linear models were estimated to examine if sleep quality was associated with MFQ1 and MFQ2, the two subjective memory scores. Finally, for those cognitive domains and subjective memory scores that yielded significant associations with Total PSQI, we analyzed each PSQI component in a separate model to ascertain which specific sleep component, if any, was associated with cognitive performance and subjective memory complaints. Statistical analyses were performed using IBM SPSS Statistics v21. Using Bonferroni correction 
Table 2. Neuropsychological measures

\begin{tabular}{|c|c|c|}
\hline Cognitive Domains & Measures & $\operatorname{MEAN}(\mathrm{SD})$ \\
\hline \multirow[t]{5}{*}{ Simple attention } & $\mathrm{T}$-score for detectability (d-prime) & $45.3(11.3)$ \\
\hline & T-score for hit reaction time & $54.8(12.2)$ \\
\hline & T-score for standard error & $49.4(10.0)$ \\
\hline & $\mathrm{T}$-score for reaction time by interstimulus interval & $54.2(9.0)$ \\
\hline & $\mathrm{T}$-score for standard error by interstimulus interval & $54.4(11.8)$ \\
\hline \multirow[t]{2}{*}{ Sustained attention } & $\mathrm{T}$-score for hit reaction time by block & $47.8(9.6)$ \\
\hline & T-score for standard error by block & $53.8(8.8)$ \\
\hline \multirow[t]{2}{*}{ Executive function $^{a}$} & Time on TMT part B - A (seconds) & $40.2(21.0)$ \\
\hline & $\mathrm{T}$-score for commission errors & $45.1(7.8)$ \\
\hline \multirow[t]{2}{*}{ Memory (Recall) } & BVMT-R (delayed score) & $8.4(2.1)$ \\
\hline & Buschke Selective Reminding Test (delayed recall) & $10.2(2.1)$ \\
\hline
\end{tabular}

Abbreviations: TMT, Trail Making Test; BVMT-R, The Brief Visual Memory Test - Revised.

For the scores in simple attention, sustained attention, and executive function, lower score indicates better performance.

${ }^{a}$ Executive function uses the average of three z-scores, time on TMT part B - A, T-score for commission errors, and T-score for hit reaction time.

for multiple comparisons, findings were considered significant at $0.0125(0.05 / 4$; two-tailed $)$ for the four objective cognitive domain scores and 0.025 (0.05/2; two-tailed) for the two MFQ scores.

\section{Results}

Of 221 participants, 15 were excluded from the analyses because they did not complete neuropsychological testing $(\mathrm{n}=7)$ or lacked PSQI scores $(\mathrm{n}=8) ; 3$ participants with outlying values on TMT-B - TMT-A (z-scores of $\geq 4$ ) were also excluded. As a result, the final sample size was 203, with a mean age of 60.4 (SD 6.5). The sample was predominantly Caucasian $(69.5 \%)$ and included a higher proportion of women $(69.0 \%)$, with a mean educational level of 16.4 (2.1) years and mean Total PSQI of 4.9 (2.9) (Table 1). Cognitive test scores are presented in Table 2.

\section{PSQI and cognitive domains}

Poorer sleep quality, as measured by Total PSQI score, was significantly associated with worse scores on sustained attention $[\mathrm{F}(1,197)=10.03, p=0.002$, partial $r=-0.22$ (Figure 1)]. The Total PSQI score was not significantly associated with simple attention, executive function, or recall memory (Table 3). ApoE4, OSA risk, gender, and age did not moderate the relationship between PSQI and cognitive domain scores (all interactions $p>0.2$ ).

Post hoc analyses examining the relationships between specific sleep components and sustained attention revealed significant correlations between sustained attention and subjective sleep quality as well as sleep disturbance. The group reporting good sleep quality $(\mathrm{n}=174)$ showed significantly better performance in sustained attention compared with the group with bad sleep quality $(\mathrm{n}=29) \quad[\mathrm{F}(1,197)=12.61, p<0.0001$, Cohen's $\mathrm{d} E S=0.72]$ (Supplement 1). The group with less sleep disturbance $(n=149)$ performed better than the group with more sleep disturbance $(\mathrm{n}=54)$ $[\mathrm{F}(1,197)=6.83, p<0.009, \mathrm{ES}=0.42]$. No significant associations between sustained attention and the other PSQI components were found.

\section{PSQI and subjective memory complaints}

Total PSQI was significantly associated with both general frequency of forgetting (MFQ1) $[\mathrm{F}(1,195)=12.11, p=0.001$, partial $\mathrm{r}=-0.24$ (Figure 2A)] and seriousness of forgetting (MFQ2), $\quad[\mathrm{F}(1,195)=9.95, \quad p=0.002, \quad$ partial $\mathrm{r}=-0.22$ (Figure 2B)] (Table 3). None of the interaction terms of PSQI total with age, gender, ApoE4, and OSA risk reached significance.

Sleep latency and daytime sleepiness were significantly associated with MFQ1 and MFQ2. Participants with sleep latency $\leq 15$ minutes $(n=87)$ reported significantly less frequency of forgetting $[\mathrm{F}(1,195)=8.7, p=0.004$, ES $=0.42]$ and less seriousness of forgetting $[\mathrm{F}(1,195)=12.95, p<0.001$, $\mathrm{ES}=0.52]$ compared to participants with sleep latency $>15$ minutes $(n=116)$. Compared to the group who reported daytime sleepiness $(n=106)$, the group with no daytime sleepiness $(\mathrm{N}=97)$ had less frequency of forgetting $[\mathrm{F}(1,195)=12.69$, $p<0.001, \mathrm{ES}=0.51]$ and less seriousness of forgetting $[\mathrm{F}(1,195)=7.87, p=0.006, \mathrm{ES}=0.40]$. Lastly, the group with less sleep disturbance $(n=149)$ reported less seriousness of forgetting $[\mathrm{F}(1,195)=6.53$, $p=0.011, \mathrm{ES}=0.41]$ (Supplement 2). 
Table 3. Association between Total PSQI and cognitive domain scores/memory self-reports

\begin{tabular}{|c|c|c|c|c|}
\hline & PARTIAL $r^{a}$ & F-statistics & $p$-VALUE & $\mathrm{SLOPE}^{\mathrm{b}}(\mathrm{SE})$ \\
\hline \multicolumn{5}{|l|}{ Cognitive domain ${ }^{c}$} \\
\hline Simple attention & 0.12 & 3.02 & 0.084 & $0.02(0.01)$ \\
\hline Sustained attention & -0.22 & 10.03 & $0.002^{\mathrm{d}}$ & $-0.06(0.02)$ \\
\hline Executive function & -0.16 & 4.91 & 0.028 & $-0.03(0.02)$ \\
\hline Recall memory & -0.04 & 0.33 & 0.568 & $-0.01(0.02)$ \\
\hline \multicolumn{5}{|l|}{ Memory self-report ${ }^{\mathrm{e}}$} \\
\hline General frequency of forgetting (MFQ1) & -0.24 & 12.11 & $0.001^{\mathrm{f}}$ & $-2.34(0.67)$ \\
\hline Seriousness of forgetting (MFQ2) & -0.22 & 9.95 & $0.002^{\mathrm{f}}$ & $-1.84(0.58)$ \\
\hline
\end{tabular}

${ }^{a}$ Adjusted for age, gender, education, and MOCA.

${ }^{\mathrm{b}}$ Unstandardized estimates.

${ }^{c} \mathrm{n}=203$; F-statistics are $\mathrm{F}(1,197)$.

${ }^{\mathrm{d}}$ Significant result, $p<0.0125(0.05 / 4)$

${ }^{\mathrm{e}} \mathrm{n}=201 ; \mathrm{F}$-statistics are $\mathrm{F}(1,195)$.

${ }^{\mathrm{f}}$ Significant result, $p<0.025(0.05 / 2)$.



Figure 1. Association between Total PSQI and sustained attention z-score.

\section{Discussion}

In this cross-sectional study of a large sample of nondemented older adults, we found that poorer sleep quality, specifically fragmented or disrupted sleep, was significantly associated with worse performance in sustained attention and increased memory complaints, and not related to simple attention and memory recall. While there was an association of sleep concerns with poorer executive function, this association did not survive the statistical correction for multiple comparisons. The ES of the significant associations ranged from 0.4 to 0.7 , indicating that they were medium to large effects and thus clinically meaningful. The sample of older adults recruited for this study had mild memory complaints, putting them at increased risk for future cognitive decline; therefore, this finding of an association between sleep and cognition in an at-risk sample is important and may serve to inform future preventive interventions that can target sleep to see if they improve cognitive functioning as well in at-risk older adults.

This association of poorer sleep and a reduction in sustained attention is consistent with a previous study indicating that as little as one night of sleep fragmentation is correlated with impaired sustained attention (Martin et al., 1996). In particular, we found that performance in sustained attention 



Figure 2. (A) Association between Total PSQI and general frequency of forgetting (MFQ1) and (B) association between Total PSQI and seriousness of forgetting (MFQ2).

measures was strongly associated with self-reported sleep quality (with a large ES of 0.7). Maintaining sustained attention involves a complex interplay of several brain regions and poor sleep quality has been associated with an increased rate of atrophy across frontal, temporal, and parietal regions (Sexton et al., 2014). Thus while the current findings are insufficient to postulate an exact mechanism of why poorer sleep and sustained attention may be related, it is plausible that the widespread cortical atrophy associated with sleep quality may be a cause of worse performance in sustained attention.
By contrast, self-reported sleep quality was not significantly associated with simple attention and memory. While there was an association between poorer sleep quality and worse executive function, this did not survive correction. In the present study, we measured executive functioning using two components: set-shifting and inhibition (Miyake et al., 2000). Previous studies have shown significant associations between set-shifting and sleep quality (Blackwell et al., 2006; 2011; Yaffe et al., 2007). Moreover, in a study of 157 healthy older adults, reports of poorer sleep quality were significantly 
associated with worse performance on tasks of working memory and set-shifting compared to reports of better sleep quality (Nebes et al., 2009). The discrepancy in findings among studies could be related to the younger age of the participants included in our study, who were on average more than 10 years younger than those in the other studies. In addition, while Blackwell et al. (2011) and Nebes et al. (2009) used the PSQI as a subjective sleep quality measure, Blackwell et al. (2006) used objective sleep measurements derived from actigraphy.

We found that subjective perceptions of memory functioning, both general frequency and seriousness of forgetting, were significantly linked to the PSQI total score, sleep latency, and daytime sleepiness. Such associations have been previously reported in older African American and Korean adults, where lengthened sleep latency was associated with severe subjective memory complaints (Gamaldo et al., 2019; Kang et al., 2017). Thus, adults with frequent memory complaints tend to report longer periods to fall asleep, or decreased sleep efficiency. Subjective memory problems also have been shown to be an early indicator of subsequent cognitive decline in older individuals (Gifford et al., 2014; Reisberg et al., 2010). Thus, our finding that sleep disturbances, even in participants with only a mild degree of self-reported sleep problems, were associated with a greater degree of subjective memory complaints is noteworthy. It is possible that those reporting poor sleep are at increased risk for future cognitive decline, and vice versa.

A strength of our study is that we assessed a broad set of neurocognitive measures in a relatively large sample, but some potential limitations need consideration when interpreting the results. Sleep quality was not evaluated using objective measures such as polysomnography or actigraphy. Examining brain activity through different sleep stages may provide more insight in terms of neurophysiological mechanisms of how sleep affects the different domains of cognitive function. Sleep quality was measured using self-reports, a common and convenient method, and previous research indicates that PSQI self-report measures correlate with objective measures of sleep (e.g. Bliwise et al., 2014). However, not all investigations show significant correlations between the PSQI self-report measure and objective sleep measures in both younger and older adults (Grandner et al., 2006). Thus, the PSQI may not be the best sleep quality measure for older adults, due to its reliance on participant's recall capacity (Landry et al., 2015). In addition, PSQI questions were designed to track sleep quality within a 1-month period (Backhaus et al., 2002) and can measure various patterns of sleep problems; however, insomnia or other sleep disorders that can affect cognition cannot be diagnosed using this assessment tool. Also, we analyzed components of sleep using categories, and may have thus not been able to detect some nuances in associations with cognition and memory self-reports; for example, not only short sleep, but long sleep (e.g. $>9$ hours) can also be associated with worse cognitive functions and the ability to detect such nonlinear relationships may have been lost by dichotomizing sleep duration. Due to the lack of available data, we were also unable to examine sleep duration as a continuous variable or examine the factor scores of PSQI in addition to the PSQI component scores.

\section{Conflict of interest}

The University of California, Los Angeles, owns a U.S. patent $(6,274,119)$ entitled "Methods for Labeling B-Amyloid Plaques and Neurofibrillary Tangles;" which has been licensed to Ceremark Pharma, LLC. Dr. Small is among the inventors and has a financial interest in Ceremark Pharma, LLC. Dr. Small also reports having served as an advisor to and/or having received lecture fees from AARP, Acadia, Allergan, Avanir, Axovant, the Gerontological Association of America, Handok, Herbalife, Lundbeck, Lilly, Genentech, Medscape, Otsuka, Recruitment Partners, Roche, and Theravalues. The other authors have no conflicts of interest to declare.

\section{Funding}

This work was supported by the Fran and Ray Stark Foundation Fund for Alzheimer's Disease Research, The Wonderful Company, LLC, John and Barbara McLoughlin Gift Fund (DM), the Parlow-Solomon Professorship (GWS), and the National Institute on Aging of the National Institutes of Health (K23AG049955 to JMD). The original study protocol was registered in ClinicalTrials .gov (Identifier: NCT02093130).

\section{Description of authors' roles}

P. Siddarth and G.W. Small designed the study. L. Ercoli and K. Miller supervised the data collection and collected the data. P. Siddarth was responsible for the statistical design of the study. $P$. Siddarth and K. Thana-udom carried out the statistical analysis. P. Siddarth wrote the paper and R. Ojha, D. Merrill, J.M. Dzierzewski, G.W. Small, and L. Ercoli assisted with writing. 


\section{Supplementary material}

To view supplementary material for this article, please visit https://doi.org/10.1017/S104161022 0003324

\section{References}

Arbuthnott, K. and Frank, J. (2000). Trail making test, part $\mathrm{B}$ as a measure of executive control: validation using a setswitching paradigm. Fournal of Clinical and Experimental Neuropsychology, 22, 518-528. https://doi.org/10.1076 /1380-3395(200008)22:4;1-0;FT518

Backhaus, J., Junghanns, K., Broocks, A., Riemann, D. and Hohagen, F. (2002). Test-retest reliability and validity of the Pittsburgh Sleep Quality Index in primary insomnia. Fournal of Psychosomatic Research, 53, 737-740. https://doi.org/10.1016/S0022-3999(02)00330-6

Bastien, C. H., Fortier-Brochu, E., Rioux, I., LeBlanc, M., Daley, M. and Morin, C. M. (2003). Cognitive performance and sleep quality in the elderly suffering from chronic insomnia. Relationship between objective and subjective measures. Fournal of Psychosomatic Research, 54, 39-49. https://doi.org/10.1016/S00223999(02)00544-5

Beck, A. T., Steer, R. A. and Brown, G. K. (1996). BDIII, Beck Depression Inventory: Manual (2nd ed.). San Antonio, TX: Psychological Corp.

Benedict R. H. D., Schretlen D., Groninger L., Dobraski, M. and Shpritz, B. (1996). Revision of the Brief Visuospatial Memory Test: studies of normal performance, reliability, and validity. Psychological Assessment, 8, 145-153. https://doi.org/10.1037/10403590.8.2.145

Blackwell, T. et al. (2011). Association of sleep characteristics and cognition in older community-dwelling men: the MrOS sleep study. Sleep, 34, 1347-1356. https:// doi.org/10.5665/SLEEP.1276

Blackwell, T. et al. (2006). Poor sleep is associated with impaired cognitive function in older women: the study of osteoporotic fractures. Fournals of Gerontology Series $A$ Biological Sciences and Medical Sciences, 61, 405-410. https:// doi.org/10.1093/gerona/61.4.405

Blackwell, T. et al. (2014). Associations of objectively and subjectively measured sleep quality with subsequent cognitive decline in older community-dwelling men: the MrOS sleep study. Sleep, 37, 655-663. https://doi.org/10 $.5665 /$ sleep. 3562

Bliwise, D. L., Holm-Larsen, T. and Goble S. (2014). Increases in duration of first uninterrupted sleep period are associated with improvements in PSQI-measured sleep quality. Sleep Medicine, 15, 1276-1278. https://doi.org/10 $.1016 /$ j.sleep.2014.05.013

Buschke, H. (1973). Selective reminding for analysis of memory and learning. Fournal of Verbal Learning and Verbal Behavior, 12, 543-550. https://doi.org/10.1016/S00225371(73)80034-9

Buysse, D. J., Reynolds, C. F., Monk, T. H., Berman, S. R. and Kupfer, D. J. (1989). The Pittsburgh Sleep Quality Index: A new instrument for psychiatric practice and research. Psychiatry Research, 28, 193-213. https://doi .org/10.1016/0165-1781(89)90047-4

Campbell, S. S. and Murphy, P. J. (2007). The nature of spontaneous sleep across adulthood. Fournal of Sleep Research, 16, 24-32 https://doi.org/10.1111/j.1365-2869 .2007.00567.x

Choe Y. M., Byun M. S., Lee J. H., Sohn, B. K., Lee, D. Y. and Kim, J. W. (2018). Subjective memory complaint as a useful tool for the early detection of Alzheimer's disease. Neuropsychiatric Disease and Treatment, 14, 2451-2460. https://doi.org/10.2147/NDT.S174517

Chung, F., Abdullah, H. R. and Liao, P. (2016). STOPBang Questionnaire: a practical approach to screen for obstructive sleep apnea. Chest, 149, 631-638. https://doi .org/10.1378/chest.15-0903

Conners, C. K. (2000). Conners' Continuous Performance Test (CPT-2) Computer Program for Windows, Technical Guide, and Software Manual. Toronto, ON: Multi Health Systems.

Crowley, K. (2011). Sleep and sleep disorders in older adults. Neuropsychology Review, 21, 41-53. https://doi.org/10.1007 /s11065-010-9154-6

Davis, H. P., Klebe, K. J., Guinther, P. M., Schroder, K. B., Cornwell, R. E. and James, L. E. (2013). Subjective organization, verbal learning, and forgetting across the life span: from 5 to 89. Experimental Aging Research, 39, 1-26. https://doi.org/10.1080/0361073X.2013.741956

Dijk, D. J., Duffy, J. F. and Czeisler, C.A. (2001). Agerelated increase in awakenings: impaired consolidation of NonREM sleep at all circadian phases. Sleep, 24, 565-577, https://doi.org/10.1093/sleep/24.5.565

Dorffner, G., Vitr, M. and Anderer P. (2015). The effects of aging on sleep architecture in healthy subjects. Advances in Experimental Medicine and Biology, 821, 93-100. doi: 10 .1007/978-3-319-08939-3_13.

Dzierzewski, J. M., Dautovich, N. and Ravyts, S. (2018). Sleep and cognition in older adults. Sleep Medicine Clinics, 13, 93-106. https://doi.org/10.1016/j.jsmc.2017.09.009

Fjell, A. M. et al. (2020). Self-reported sleep relates to hippocampal atrophy across the adult lifespan: results from the Lifebrain consortium. Sleep, 43, zsz280. https://doi .org/10.1093/sleep/zsz280

Foley, D. J., Masaki, K., White, L., Larkin, E. K., Monjan, A. and Redline, S. (2003). Sleep- disordered breathing and cognitive impairment in elderly JapaneseAmerican men. Sleep, 26, 596-599. https://doi.org/10.1093 /sleep/26.5.596

Fortenbaugh, F. C., DeGutis, J. and Esterman, $M$. (2017). Recent theoretical, neural, and clinical advances in sustained attention research. Annals of New York Academy Sciences, 1396, 70-91. https://doi.org/10.1111/nyas.13318

Gamaldo, A. A., Wright, R. S., Aiken-Morgan, A. T, Allaire, J. C., Thorpe, R. J. Jr. and Whitfield, K. E. (2019). The Association between Subjective Memory Complaints and Sleep within Older African American Adults. Fournals of Gerontology Series B Psychological Sciences and Social Sciences, 74, 202-211. https://doi.org/10 $.1093 /$ geronb/gbx069

Gifford, K. A. et al. (2014). The source of cognitive complaints predicts diagnostic conversion differentially among nondemented older adults. Alzheimers and Dementia, 10, 319-327. https://doi.org/10.1016/j.jalz.2013.02.007 
Gilewski, M. J., Zelinski, E. M. and Schaie, K. W. (1990). The Memory Functioning Questionnaire for assessment of memory complaints in adulthood and old age. Psychology and Aging, 5, 482-490. https://doi.org/10.1037//0882-7974 .5 .4 .482

Glisky, E. L. (2007). Changes in Cognitive Function in Human Aging. In: Riddle, D.R. (Eds.), Brain Aging: Models, Methods, and Mechanisms. Boca Raton (FL): CRC Press/ Taylor \& Francis.

Gosselin, N., Baril, A. A., Osorio, R. S., Kaminska, M. and Carrier, J. (2019). Obstructive sleep apnea and the risk of cognitive decline in older adults. American fournal of Respiratory and the Critical Care Medicine, 199, 142-148 https://doi.org/10.1164/rccm.201801-0204PP

Grandner, M. A., Kripke, D. F., Yoon, I. Y. and Youngstedt, S. D. (2006). Criterion validity of the Pittsburgh Sleep Quality Index: investigation in a nonclinical sample. Sleep and Biological Rhythms, 4, 129-139. https://doi.org/10.1111/j.1479-8425.2006.00207.x

Jessen, F. (2010). Prediction of dementia by subjective memory impairment. Archives of General Psychiatry, 67, 414. https://doi.org/10.1001/archgenpsychiatry.2010.30

Kang, S.-H. et al. (2017). Subjective memory complaints in an elderly population with poor sleep quality. Aging and Mental Health, 21, 532-536. https://doi.org/10.1080 /13607863.2015.1124839

Krishnan, V. and Collop, N. A. (2006). Gender differences in sleep disorders. Current Opinion in Pulmonary Medicine, 12, 383-389. https://doi.org/10.1097/01.mcp $.0000245705 .69440 .6 \mathrm{a}$

Landry, G. J., Best, J. R. and Liu-Ambrose, T. (2015). Measuring sleep quality in older adults: a comparison using subjective and objective methods. Frontiers in Aging Neuroscience, 7, 166. doi: 10.3389/fnagi.2015.00166

Lim, A. S., Kowgier, M., Yu, L., Buchman, A. S. and Bennett, D. A. (2013). Sleep fragmentation and the risk of incident Alzheimer's disease and cognitive decline in older persons. Sleep, 36, 1027-1032. https://doi.org/10.5665/sleep .2802

Lim, A. S., Yu, L., Kowgier, M., Schneider, J. A., Buchman, A. S. and Bennett, D. A. (2013). Sleep modifies the relation of APOE to the risk of Alzheimer disease and neurofibrillary tangle pathology. $\mathcal{F} A M A$ Neurology, 70. doi: 10.1001/jamaneurol .2013 .4215

Lo, J. C., Loh, K. K., Zheng, H., Sim, S. K. Y. and Chee, M. W. L. (2014). Sleep duration and age-related changes in brain structure and cognitive performance. Sleep, 37, 1171-1178. https://doi.org/10.5665/sleep.3832

Loerbroks, A., Debling, D., Amelang, M. and Stürmer, T. (2010). Nocturnal sleep duration and cognitive impairment in a population-based study of older adults. International fournal of Geriatric Psychiatry, 25, 100-109. https://doi.org/10.1002/gps.2305

Martin, S. E., Engleman, H. M., Deary, I. J. and Douglas, N.J. (1996). The effect of sleep fragmentation on daytime function. American fournal of Respiratory and Critical Care Medicine, 153, 1328-1332. https://doi.org/10 .1164/ajrccm.153.4.8616562

McSorley, V. E., Sun Bin, Y. and Lauderdale, D. S. (2019). Associations of sleep characteristics with cognitive function and decline among older adults. American fournal of Epidemiology, 188, 1066-1075. https://doi.org/10.1093 /aje/kwz037

Miyake, A., Friedman, N. P., Emerson, M. J., Witzki, A. H., Howerter, A. and Wager, T. D. (2000). The unity and diversity of executive functions and their contributions to complex "frontal lobe" tasks. Cognitive Psychology, 41, 49-100. https://doi.org/10.1006/cogp.1999.0734

Moore, P., Bardwell, W. A., Ancoli-Israel, S. and Dimsdale, J.E. (2001). Association between polysomnographic sleep measures and health-related quality of life in obstructive sleep apnea. Fournal of Sleep Research, 10, 303-308. https://doi.org/10.1046/j.13652869.2001.00264.x

Nasreddine, Z. S. et al. (2005). The Montreal Cognitive Assessment, MoCA: A brief screening tool for mild cognitive impairment. Fournal of the American Geriatrics Society, 53, 695-699. https://doi.org/10.1111/j.1532-5415 .2005.53221.x

Nebes, R. D., Buysse, D. J., Halligan, E. M., Houck, P. R. and Monk, T. H. (2009). Self-reported sleep quality predicts poor cognitive performance in healthy older adults. Fournal of Gerontology Series B Psychological Sciences and Social Sciences, 64, 180-187. doi: 10.1093/geronb /gbn037

Ohayon, M. M., Carskadon, M. A., Guilleminault, C. and Vitiello, M. V. (2004). Mata-analysis of quantitative sleep parameters from childhood to old age in healthy individuals: developing normative sleep values across the human lifespan. Sleep, 27, 1255-1273. https://doi.org/10 $.1093 /$ sleep/27.7.1255

Reisberg, B., Shulman, B., Torossian, C., Leng, L. and Zhu, W. (2010). Outcome over seven years of healthy adults with and without subjective cognitive impairment. Alzheimers and Dementia, 6, 11-24. https://doi.org/10.1016/j .jalz.2009.10.002

Rozzini, L. et al. (2018). Non-amnestic mild cognitive impairment and sleep complaints: a bidirectional relationship? Aging Clinical and Experimental Research, 30, 661-668. https://doi.org/10.1007/s40520-017-0814-8

Saint Martin, M., Sforza, E., Barthélémy, J. C., Thomas-Anterion, C. and Roche, F. (2012). Does subjective sleep affect cognitive function in healthy elderly subjects? The Proof cohort. Sleep Medicine, 13, 1146-1152. https://doi.org/10.1016/j.sleep.2012.06.021

Salthouse, T. (2010). Influence of age on practice effects in longitudinal neurocognitive change. Neuropsychology, 24, 563. https://doi.org/10.1037/a0019026

Scullin, M. K. and Bliwise, D. L. (2015). Sleep, cognition, and normal aging. Perspectives on Psychological Science, 10, 97-137. https://doi.org/10.1177/1745691614556680

Sexton, C. E., Storsve, A. B. and Walhovd, K. B. (2014). Poor sleep quality is associated with increased cortical atrophy in community-dwelling adults. Neurology, 83, 967-973. https://doi.org/10.1212/WNL .0000000000000774

Siddarth, P. et al. (2020). Randomized placebo-controlled study of the memory effects of pomegranate juice in middleaged and older adults. American fournal of Clinical Nutrition, 111, 170-177. https://doi.org/10.1093/ajcn /nqz241

Tsapanou, A. et al. (2017). Sleep quality and duration in relation to memory in the elderly: Initial results from the 
Hellenic Longitudinal Investigation of Aging and Diet. Neurobiology of Learning and Memory, 141, 217-225. https:// doi.org/10.1016/j.nlm.2017.04.011

Vance, D. E. (2012). Potential factors that may promote successful cognitive aging. Nursing: Research and Reviews, 2, 27-32. https://doi.org/10.2147/NRR.S32229

Waters, F. and Bucks, R. S. (2011). Neuropsychological effects of sleep loss: Implication for neuropsychologists. Fournal of the International Neuropsychological Society, 17, 571-586. https://doi.org/10.1017/S1355617711000610

Wolf, P. A., D'Agostino, R. B., Belanger, A. J. and Kannel, W. B. (1991). Probability of stroke: A risk profile from the Framingham Study. Stroke, 22, 312-318. https:/ doi.org/10.1161/01.STR.22.3.312

Wolkove, N., Elkholy, O., Baltzan, M. and Palayew, M. (2007). Sleep and aging: 1 . Sleep disorders commonly found in older people. Canadian Medical Association fournal, 176, 1299-1304. https://doi.org/10.1503/cmaj.060792

Yaffe, K. et al. (2007). Preclinical cognitive decline and subsequent sleep disturbance in older women. Neurology, 69, 237-242. https://doi.org/10.1212/01.wnl.0000265814 $.69163 . \mathrm{da}$

Yu, J., Mahendran, R., Rawtaer, I., Kua E. H. and Feng, L. (2017). Poor sleep quality is observed in mild cognitive impairment and is largely unrelated to depression and anxiety. Aging $\mathcal{E}$ Mental Health, 21, 823-828. https://doi .org/10.1080/13607863.2016.1161007

Zelinski, E. M., Gilewski, M. J. and Anthony-Bergstone, C. R. (1990). Memory Functioning Questionnaire: Concurrent validity with memory performance and selfreported memory failures. Psychology and Aging, 5, 388-399. https://psycnet.apa.org/doi/10.1037/0882-7974.5.3.388 